\title{
DETECTING ASYMPTOTIC NON-REGULAR VALUES BY POLAR CURVES
}

\author{
ZBIGNIEW JELONEK AND MIHAI TIBĂR
}

\begin{abstract}
We locate the Malgrange non-regular values of a given polynomial function $f: \mathbb{C}^{n} \rightarrow \mathbb{C}$ by using a series of affine polar curves. We moreover show that all non-trivial Malgrange non-regular values of $f$ are indicated by a single "super-polar curve" which we introduce here, providing also an effective algorithm of detection.
\end{abstract}

\section{INTRODUCTION}

Let $f: \mathbb{C}^{n} \rightarrow \mathbb{C}$ be a polynomial of degree $d \geq 2$. René Thom proved that $f$ is a $C^{\infty}$-fibration outside a finite set, where the smallest such set is called the bifurcation set of $f$ and is denoted by $B(f)$. Roughly speaking the set $B_{\infty}(f)$ consists of points at which $f$ is not a locally trivial fibration at infinity (i.e., outside a large ball). Two fundamental questions appear in a natural way: how to characterize the set $B(f)$ and how to estimate the number of points of this set.

Let us recall that the set $B(f)$ contains the set $f(\operatorname{Sing} f)$ of critical values of $f$ and the set $B_{\infty}(f)$ of bifurcations points at infinity.

In case $n=2$ there are well-known criteria to detect $B(f)$, see e.g. [Ti4], [Du], and there are also estimations of the upper bound of the number $\# B(f)$ in terms of the degree or other data [Ha1], [Ha2], [Jel6], [LO], [Gw], [JT] etc.

Whenever $n>2$ one has no exact criteria but one defines regularity conditions at infinity that each yield some finite set of values containing $B(f)$ and thus approaching the problem of estimating $\# B(f)$. To control the set $B_{\infty}(f)$ one can use the set of asymptotic critical values of $f$ :

$$
K_{\infty}(f):=\left\{y \in \mathbb{C} \mid \exists\left(x_{l}\right)_{l \in \mathbb{N}},\left\|x_{l}\right\| \rightarrow \infty \text {, such that } f\left(x_{l}\right) \rightarrow y \text { and }\left\|x_{l}\right\|\left\|d f\left(x_{l}\right)\right\| \rightarrow 0\right\} .
$$

If $c \notin K_{\infty}(f)$, then it is usual to say that $f$ satisfies Malgrange's condition at $c$ ( or $c$ is Malgrange regular). The set $K_{\infty}(f)$ naturally decomposes into two pieces: the set $T K_{\infty}(f)$ of trivial Malgrange non-regular values which come from the critical points of $f$ (i.e. there is a sequence $x_{l} \rightarrow \infty$ such that $x_{l} \in \operatorname{Sing}(f)$ and $f\left(x_{l}\right) \rightarrow y$ ) and the remaining set $N K_{\infty}(f):=K_{\infty}(f) \backslash T K_{\infty}(f)$ of non-trival Malgrange non-regular values. Of course $T K_{\infty}(f) \subset f(\operatorname{Sin} g f)$. Since the set $f(\operatorname{Sing} f)$ is relatively easy to compute, the problem which remains is how to compute the set $N K_{\infty}(f)$.

Date: July 17, 2018.

2010 Mathematics Subject Classification. 32S05, 32S50, 14D06 14Q20, 58K05, 14P10.

Key words and phrases. polar curve, bifurcation locus, Malgrange non-regular values, effectivity.

The authors acknowledge the support of the Labex CEMPI (ANR-11-LABX-0007-01) at Lille, and of the CIRM at Luminy through the RIP program. The first author was partially supported by the NCN grant 2013/09/B/ST1/04162, 2014-2017. 
It was proved (cf [Pa2], [JK1], [Jel4]) that one has the inclusion $B_{\infty}(f) \subset K_{\infty}(f)$. Setting $K(f):=f(\operatorname{Sing} f) \cup K_{\infty}(f)$, we get the inclusion $B(f) \subset K(f)$. Estimations of the number of the Malgrange non-regular values have been given in [JK1]. An algorithmic method for recovering the set $K_{\infty}(f)$ has been produced more recently [JK2].

We present here two new methods for detecting $K_{\infty}(f)$ and for estimating the number $\# K_{\infty}(f)$, together with an effective algorithm. Our first approach, based on the use of a series of polar curves and their relation to Malgrange non-regularity via the $t$-regularity, yields an exact characterisation of the set $N K_{\infty}(f)$. We shall recall the notions and the relevant preliminary results in $\S 2$, but let us introduce already here our first main statement.

Let $\left\{x_{1}, \ldots, x_{n}\right\}$ be a generic system of coordinates of $\mathbb{C}^{n}$, after Definition 2.8. Let us consider the successive restrictions of $f$ to the affine hyperplanes:

$$
f_{0}:=f, \quad f_{1}:=f_{\mid x_{1}=0}, \ldots, f_{n-2}:=f_{\mid x_{1}=\cdots=x_{n-2}=0},
$$

and the corresponding generic polar curves $\Gamma\left(x_{i}, f_{i-1}\right)$, for $i=1, \ldots, n-1$.

For a mapping $g: X \rightarrow Y$, let $J_{g}$ denote the non-properness set [Jel1], [Jel2] (also called the Jelonek set) of the mapping $g$, see $\S 4$, Theorem 4.1. If $A \subset X$, then by $J_{g}(A)$ we denote the non-properness set of the restriction $g_{\mid A}$.

We say that an irreducible algebraic variety $S \subset \mathbb{C}^{n}$ is horizontal if $f(S)$ is not a point (i.e. $S$ is not included in some fibre of $f$ ). The union of all horizontal components of the polar curve $\Gamma\left(x_{i}, f_{i-1}\right)$ will be called the horizontal part and will be denoted by $H \Gamma\left(x_{i}, f_{i-1}\right)$.

We prove the following characterisation of the set $N K_{\infty}(f)$ :

Theorem 1.1. The set $N K_{\infty}(f)$ of non-trivial Malgrange non-regular regular values of $f$ is included in the union of the non-properness sets of the mapping $f$ restricted to a horizontal part of the polar curves $\Gamma\left(x_{i}, f_{i-1}\right)$, more precisely we have the equality:

$$
N K_{\infty}(f)=\bigcup_{i=1}^{n-1} J_{f}\left(H \Gamma\left(x_{i}, f_{i-1}\right)\right) \backslash J_{f}(\operatorname{Sing} f) .
$$

Note that $J_{f}(\operatorname{Sing} f)$ equals the the set of critical values of $f$ which are images of fibers containing nonisolated singularities.

Let Sing $f=S_{0} \cup S_{1} \cup \cdots \cup S_{r}$ be the decomposition of the singular locus into irreducible components, where $S_{0}$ denotes the union of all point-components (i.e. $S_{0}$ is the set of isolated singularities of $f$ ). For $i>1$ we denote by $d_{i}=\operatorname{deg} S_{i}$ the degree of the positive dimensional component $S_{i}$.

Corollary 1.2. For $d>2$ we have:

$$
\# N K_{\infty}(f) \leq \frac{(d-1)^{n}-1}{d-2}-\sum_{i=1}^{r} d_{i} \operatorname{dim} S_{i},
$$

and for $d=2$ :

$$
\# N K_{\infty}(f) \leq n-1-\sum_{i=1}^{r} d_{i} \operatorname{dim} S_{i}
$$


According to their definition, the polar curves of the above statement are affine curves, some of them are maybe empty, and they do not detect, in general, values from $T K_{\infty}(f)$. The first polar curve $\Gamma\left(x_{1}, f\right)$ detects some Malgrange non-regular value $c \in N K_{\infty}(f)$ whenever the fiber $f^{-1}(c)$ has only isolated singularities at infinity in the sense of Definition 2.5, cf Theorem 2.9. However, the first polar curve may not detect all values from $N K_{\infty}(f)$ and that is why we need more polar curves. We explain this phenomenon by the existence of non-isolated t-singularities at infinity, cf $\S 3$. For example, if $f(x, y, x)=x+x^{2} y$ then the polar curve of $f$ is empty, but $f$ has a non-trivial Malgrange non-regular value 0 . This example also shows that Theorem 3.6 in [Sa] is not correct. More precisely, if we use polar curves, then the problem of detecting non-trivial Malgrange non-regular values cannot be done in a single step (as was wrongly claimed in [Sa]), but turns out to fall into $n-1$ steps as we describe now in our Theorem 1.1, each step being the detection of the non-properness set of a certain generic polar curve.

However, the question "is it possible to recover all non-trivial Malgrange non-regular values in just one single step" subsists as a chalenging problem. We solve it positively in the second part of our paper by introducing a new and different device called "superpolar curve". Let us give here an outline of its construction. We consider the following polynomials:

$$
g_{i}(a, b)=\sum_{j} a_{i j} \frac{\partial f}{\partial x_{j}}+\sum_{j, k} b_{i j k} x_{k} \frac{\partial f}{\partial x_{j}}, i=1, \ldots, n-1,
$$

where $a_{i j}, b_{i j k}$ are complex constants. Let:

$$
\Gamma_{f}(a, b):=\text { closure }\left\{V\left(g_{1}, \ldots, g_{n-1}\right) \backslash \operatorname{Sing}(f)\right\},
$$

where we use here the Zariski closure. It turns out that, for general $a_{i j}, b_{i j k}$ the set $\Gamma_{f}(a, b)$ is a non-empty curve, which we shall call super-polar curve of $f$. We say that a component $S \subset \Gamma_{f}(a, b)$ is horizontal if $f(S)$ is not a single point. The union of all horizontal components of $\Gamma_{f}(a, b)$ will be called the horizontal part of $\Gamma_{f}(a, b)$ and will be denoted by $H \Gamma_{f}(a, b)$. We obviously have the inclusion $J_{f}\left(H \Gamma_{f}(a, b)\right) \subset J_{f}\left(\Gamma_{f}(a, b)\right)$. We prove the following result:

Theorem 1.3. The set $N K_{\infty}(f)$ of nontrivial Malgrange non-regular values of $f$ is included in the non-properness set of a mapping $f$ restricted to the horizontal part of a sufficiently general super-polar curve $\Gamma_{f}(a, b)$, namely one has the following inclusion:

$$
N K_{\infty}(f) \subset J_{f}\left(H \Gamma_{f}(a, b)\right) .
$$

Corollary 1.4. If $n>2$ and $N K_{\infty}(f) \neq \emptyset$ then:

$$
\# N K_{\infty}(f) \leq d^{n-1}-1-\sum_{i=1}^{r} d_{i} .
$$

In particular if $N K_{\infty}(f) \neq \emptyset$, then:

$$
\# K_{\infty}(f) \leq d^{n-1}-1-\sum_{i=1}^{r}\left(d_{i}-1\right) .
$$


In case $n=2$, if $N K_{\infty}(f) \neq \emptyset$, then:

$$
\# N K_{\infty}(f) \leq d-2-\sum_{i=1}^{r} d_{i}, \quad \text { and } \quad \# K_{\infty}(f) \leq d-2-\sum_{i=1}^{r}\left(d_{i}-1\right) .
$$

The plan of the paper goes as follows: in $\S 2$ and $\S 4$ we develop some preliminary results in order to prepare the proofs of Theorem 1.1 in $\S 3$, and of Theorem 1.3 in $\S 5$, together with their corollaries, respectively. In $\S 6$ we sketch the algorithm to detect the set $N K_{\infty}(f)$ effectively.

\section{REgularity CONDitions AT INFINITY}

2.1. Malgrange regularity condition at a point at infinity. Pham formulated in $[\mathrm{Ph}, 2.1]$ a regularity condition which he attributed to Malgrange. We recall the localized version at infinity, after [Ti2], [Ti4].

We identify $\mathbb{C}^{n}$ to the graph of $f$, namely $X:=\left\{(x, \tau) \in \mathbb{C}^{n} \times \mathbb{C} \mid f(x)=\tau\right\}$, and consider its algebraic closure in $\mathbb{P}^{n} \times \mathbb{C}$, which is the hypersurface:

$$
\mathbb{X}=\left\{\tilde{f}\left(x_{0}, x\right)-\tau x_{0}^{d}=0\right\} \subset \mathbb{P}^{n} \times \mathbb{C},
$$

where $x_{0}$ denotes the variable at infinity, $d=\operatorname{deg} f$ and $\tilde{f}\left(x_{0}, x\right)$ denotes the homogenization of degree $d$ of $f$ by the variable $x_{0}$. Let $\boldsymbol{t}: \mathbb{X} \rightarrow \mathbb{C}$ denote the restriction to $\mathbb{X}$ of the second projection $\mathbb{P}^{n} \times \mathbb{C} \rightarrow \mathbb{C}$, a proper extension of the map $f$. We denote by $\mathbb{X}^{\infty}=\mathbb{X} \backslash X$ the divisor at infinity defined in each affine chart by the equation $x_{0}=0$.

Definition 2.1. [Ti4] Let $\left\{\mathbf{x}_{i}\right\}_{i \in \mathbb{N}} \subset \mathbb{C}^{n}$ be a sequence of points with the following properties:

$\left(\mathrm{L}_{1}\right) \quad\left\|\mathbf{x}_{i}\right\| \rightarrow \infty$ and $f\left(\mathbf{x}_{i}\right) \rightarrow \tau$, as $i \rightarrow \infty$.

$\left(\mathrm{L}_{2}\right) \quad \mathbf{x}_{i} \rightarrow p \in \mathbb{X}^{\infty}$, as $i \rightarrow \infty$.

One says that the fibre $f^{-1}(\tau)$ verifies the Malgrange condition if there is $\delta>0$ such that, for any sequence of points with property $\left(\mathrm{L}_{1}\right)$ one has

(M) $\quad\left\|\mathbf{x}_{i}\right\| \cdot\left\|\operatorname{grad} f\left(\mathbf{x}_{i}\right)\right\|>\delta$.

We say that $f$ verifies Malgrange condition at $p \in \mathbb{X}^{\infty}$ if there is $\delta_{p}>0$ such that one has (M) for any sequence of points with property $\left(\mathrm{L}_{2}\right)$.

REMARK 2.2. It follows from the definition that $f^{-1}(\tau)$ verifies the Malgrange condition if and only if $f$ verifies Malgrange condition (M) at any point $p=(z, \tau) \in \mathbb{X}^{\infty} \cap \boldsymbol{t}^{-1}(\tau)$ and for the same positive constant $\delta_{p}=\delta$.

2.2. Characteristic covectors and $t$-regularity. We recall the notion of $t$-regularity from [Ti1], [Ti4]. Let $H^{\infty}=\left\{\left[x_{0}: x_{1}: \ldots: x_{n}\right] \in \mathbb{P}^{n} \mid x_{0}=0\right\}$ denote the hyperplane at infinity and let $\mathbb{X}^{\infty}:=\mathbb{X} \cap\left(H^{\infty} \times \mathbb{C}\right)$.

We consider the affine charts $U_{j} \times \mathbb{C}$ of $\mathbb{P}^{n} \times \mathbb{C}$, where $U_{j}=\left\{x_{j} \neq 0\right\}, j=0,1, \ldots, n$. Identifying the chart $U_{0}$ with the affine space $\mathbb{C}^{n}$, we have $\mathbb{X} \cap\left(U_{0} \times \mathbb{C}\right)=\mathbb{X} \backslash \mathbb{X}^{\infty}=X$ and $\mathbb{X}^{\infty}$ is covered by the charts $U_{1} \times \mathbb{C}, \ldots, U_{n} \times \mathbb{C}$.

If $g$ denotes the projection to the variable $x_{0}$ in some affine chart $U_{j} \times \mathbb{C}$, then the relative conormal $C_{g}\left(\mathbb{X} \backslash \mathbb{X}^{\infty} \cap U_{j} \times \mathbb{C}\right) \subset \mathbb{X} \times \check{\mathbb{P}}^{n}$ is well defined (see e.g. [Ti3], [Ti5]), with the projection $\pi(y, H)=y$, where $\check{\mathbb{P}}^{n}$ is identified to the projective space of hyperplanes 
in $U_{j} \times \mathbb{C}$. Let us then consider the space $\pi^{-1}\left(\mathbb{X}^{\infty}\right)$ which is well defined for every chart $U_{j} \times \mathbb{C}$ as a subset of $C_{g}\left(\mathbb{X} \backslash \mathbb{X}^{\infty} \cap U_{j} \times \mathbb{C}\right)$. By [Ti2, Lemma 3.3], the definitions coincide at the intersections of the charts.

Definition 2.3. We call space of characteristic covectors at infinity the well-defined set $\mathcal{C}^{\infty}:=\pi^{-1}\left(\mathbb{X}^{\infty}\right)$. For some $p_{0} \in \mathbb{X}^{\infty}$, we denote $\mathcal{C}_{p_{0}}^{\infty}:=\pi^{-1}\left(p_{0}\right)$.

Considering now the second projection $\boldsymbol{t}: \mathbb{P}^{n} \times \mathbb{C} \rightarrow \mathbb{C}$ in place of the function $g$ in the above consideration, we obtain the relative conormal space $C_{\boldsymbol{t}}\left(\mathbb{P}^{n} \times \mathbb{C}\right)$. Then we have:

Definition 2.4. [Ti2] We say that $f$ is t-regular at $p_{0} \in \mathbb{X}^{\infty}$ if $C_{\boldsymbol{t}}\left(\mathbb{P}^{n} \times \mathbb{C}\right) \cap \mathcal{C}_{p_{0}}^{\infty}=\emptyset$ or, equivalently, $\mathrm{d} \boldsymbol{t} \notin \mathcal{C}_{p_{0}}^{\infty}$.

Definition 2.5. We say that $f$ has isolated $t$-singularities at infinity at the fibre $f^{-1}\left(t_{0}\right)$ if this fibre has isolated singularities in $\mathbb{C}^{n}$ and if the set

$$
\operatorname{Sing}^{\infty} f:=\left\{p \in \mathbb{X}^{\infty} \mid f^{-1}\left(t_{0}\right) \text { is not } t \text {-regular at } p\right\}
$$

is a finite set.

It follows from the definition that $\operatorname{Sing}^{\infty} f$ is a closed algebraic subset of $\mathbb{X}^{\infty}$, see e.g. [Ti2], [Ti5], [DRT, §6.1]. By the algebraic Sard Theorem, the image $\boldsymbol{t}\left(\operatorname{Sing}^{\infty} f\right)$ consists of a finite number of points.

We need the following key equivalence in the localized setting (proved initially in [ST, Proposition 5.5] and [Pa1, Theorem 1.3], as explained in [Ti4]):

Theorem 2.6. [Ti5, Prop. 1.3.2] A polynomial $f: \mathbb{C}^{n} \rightarrow \mathbb{C}$ is t-regular at $p_{0} \in \mathbb{X}^{\infty}$ if and only if $f$ verifies the Malgrange condition at this point.

More precisely we have the following relations, cf [Ti3], [Ti5]:

(6) Malgrange regularity $\Longleftrightarrow t$-regularity $\Longrightarrow \rho_{E}$-regularity $\Longrightarrow$ topological triviality

which also extend to polynomial maps $\mathbb{C}^{n} \rightarrow \mathbb{C}^{p}$ as shown in $[\mathrm{DRT}]$.

2.3. Polar curves and t-regularity. We define the affine polar curves of $f$ and show how they are related to the $t$-regularity condition, after [Ti3].

Given a polynomial $f: \mathbb{C}^{n} \rightarrow \mathbb{C}$ and a linear function $l: \mathbb{C}^{n} \rightarrow \mathbb{C}$, the polar curve of $f$ with respect to $l$, denoted by $\Gamma(l, f)$, is the closure in $\mathbb{C}^{n}$ of the set $\operatorname{Sing}(l, f) \backslash \operatorname{Sing} f$, where $\operatorname{Sing}(l, f)$ is the critical locus of the map $(l, f): \mathbb{C}^{n} \rightarrow \mathbb{C}^{2}$. Denoting by $l_{H}: \mathbb{C}^{n} \rightarrow \mathbb{C}$ the unique linear form (up to multiplication by a constant) which defines a hyperplane $H \in \mathbb{C}^{n}$ (also regarded as a point in the projective space $\check{\mathbb{P}}^{n-1}$ of linear hyperplanes in $\left.\mathbb{C}^{n}\right)$, we have the following genericity result of Bertini-type.

Lemma 2.7. [Ti2, Lemma 1.4]

There exists a Zariski-open set $\Omega_{f, a} \subset \check{\mathbb{P}}^{n-1}$ such that, for any $H \in \Omega_{f, a}$ and some fixed $a \in \mathbb{C}$, the polar set $\Gamma\left(l_{H}, f\right)$ is a curve or it is an empty set, and no component is contained in the fibre $f^{-1}(a)$.

Definition 2.8. For $H \in \Omega_{f, a}$, we call $\Gamma\left(l_{H}, f\right)$ the generic affine polar curve of $f$ with respect to $l_{H}$. A system of coordinates $\left(x_{1}, \ldots, x_{n}\right)$ in $\mathbb{C}^{n}$ is called generic with respect to $f$ iff $\left\{x_{i}=0\right\} \in \Omega_{f, a}, \forall i$. 
It follows from Lemma 2.7 that such systems of coordinates are generic among all linear systems of coordinates.

Let $\overline{\Gamma\left(l_{H}, f\right)}$ and $\overline{\text { Sing } f}$ denote the algebraic closure in $\mathbb{X}$ of the respective sets. We then have:

Theorem 2.9. Let $f: \mathbb{C}^{n} \rightarrow \mathbb{C}$ be a polynomial function and let $p \in \mathbb{X} \infty, a:=\boldsymbol{t}(p)$.

(a) If $p$ is a t-regular point then $\left.p \notin \overline{\left(\Gamma\left(l_{H}, f\right)\right.} \cup \overline{\operatorname{Sing} f}\right) \cap \mathbb{X}^{\infty}$, for any $H \in \Omega_{f, a}$.

(b) Let $p$ be either t-regular or an isolated t-singularity at infinity. Then $p$ is a $t$ singularity at infinity if and only if $p \in \overline{\Gamma\left(l_{H}, f\right)}$ for some $H \in \Omega_{f, a}$.

Proof. The result and its proof can be actually extracted from [Ti5, §2.1]. More precisely: (a) follows from [Ti5, Prop. 2.1.3] and [Ti5, (2.1), pag.17].

(b) follows by combining Thm. 2.1.7, Thm. 2.1.6 and Prop. 2.1.3 from [Ti5, §2.1].

The above theorem means that isolated $t$-singularities at infinity are precisely detected by the horizontal part of the generic polar curve. In case $p \in \mathbb{X}^{\infty}$ is a non-isolated $t$ singularity (which occurs whenever $n>2$ ), the general affine polar curve $\Gamma\left(l_{H}, f\right)$ might not contain the chosen point $p$ in its closure at infinity. We shall show in the next section how to deal with this situation.

\section{Proof of Theorem 1.1}

Let $B:=\operatorname{Sing}^{\infty} f \cap\left(\mathbb{X} \backslash \cup_{a \in f(\operatorname{Sing} f)} \mathbb{X}_{a}\right)$. By Theorem 2.6, we have the equality:

$$
N K_{\infty}(f)=\boldsymbol{t}(B) .
$$

By Theorem 2.9(a) and Theorem 2.6, if the generic polar curve $\Gamma\left(l_{H}, f\right)$ is nonempty, then it intersects the hypersurface $\mathbb{X}^{\infty}$ at finitely many points and these points are $t$ singularities, hence Malgrange non-regular points at infinity.

Let us first assume that $\operatorname{dim} B=0$. Then, by Theorem 2.9, for $p \in B$ (which by our assumption is an isolated $t$-singularity), the generic polar curve passes through $p$, so this point is "detected" by the horizontal part of the polar curve $\overline{\Gamma\left(x_{1}, f\right)}$, for some generic choice of the coordinate $x_{1}$ (in the sense of Definition 2.8 and Lemma 2.7). Therefore, in the notations of the Introduction, the corresponding asymptotic non-regular value belongs to $J_{f}\left(H \Gamma\left(x_{1}, f\right)\right)$.

Therefore, in our case $\operatorname{dim} B=0$, the equality (1) follows from Theorem 2.9 and Theorem 2.6.

Let us now treat the case $\operatorname{dim} B>0$. We will show (1) by a double inclusion.

The inclusion " $\supset$ ". Let us first prove the inclusion $J_{f}\left(H \Gamma\left(x_{i}, f_{i-1}\right)\right) \backslash J_{f}(\operatorname{Sing} f) \subset$ $N K_{\infty}(f)$ for each $i=1, \ldots, n-1$. We proceed by a "reductio ad absurdum" argument. Assume that $a \notin N K_{\infty}(f)$ and denote $\mathbb{X}_{a}^{\infty}:=\mathbb{X}^{\infty} \cap \boldsymbol{t}^{-1}(a)$.

(a). If $a \in J_{f}\left(H \Gamma\left(x_{1}, f_{0}\right)\right) \backslash J_{f}(\operatorname{Sing} f)$, then there exist points $p \in \mathbb{X}_{a}^{\infty} \cap \overline{\Gamma\left(x_{1}, f_{0}\right)}$. By Theorem 2.9(a), this means that $p$ is a $t$-non-regular point, which implies in turn that $a \in N K_{\infty}(f)$, by Theorem 2.6.

(b). Assume that $a \notin J_{f}\left(H \Gamma\left(x_{i}, f_{i-1}\right)\right) \backslash J_{f}(\operatorname{Sing} f)$ for $i=1, \ldots, k-1$ (for some $k \geq 2$ ), and that $a \in J_{f}\left(H \Gamma\left(x_{k}, f_{k-1}\right)\right) \backslash J_{f}(\operatorname{Sing} f)$. 
We endow the hypersurface $\mathbb{X} \subset \mathbb{P}^{n} \times \mathbb{C}$ with a finite complex Whitney stratification $\mathcal{W}$ such that $\mathbb{X}^{\infty}:=\left\{f_{d}=0\right\} \times \mathbb{C}$ is a union of strata. Our Whitney stratification at infinity is also Thom $\left(\mathrm{a}_{x_{0}}\right)$-regular, by [Ti2, Theorem 2.9], where $x_{0}=0$ is some local equation for $H^{\infty}$ at $p$.

There exists a Zariski-open set $\Omega^{\prime} \subset \check{\mathbb{P}}^{n-1}$ of linear forms $\mathbb{C}^{n} \rightarrow \mathbb{C}$ such that, if $H \in \Omega^{\prime}$, then $\left(H^{\infty} \cap \bar{H}\right) \times \mathbb{C}$ is transversal in $H^{\infty} \times \mathbb{C}$ to all strata of $\mathcal{W}$ in the neighbourhood of $\mathbb{X}_{a}^{\infty}$. Due to the Thom $\left(\mathrm{a}_{x_{0}}\right)$-regularity of the stratification, it follows that slicing by $H \in \Omega^{\prime}$ insures the $t$-regularity of the restriction $f_{\mid H}$ at any point $p \in(\bar{H} \times \mathbb{C}) \cap \mathbb{X}_{a}^{\infty}$. More precisely, from our hypothesis $\mathrm{d} \boldsymbol{t} \notin \mathcal{C}_{p}^{\infty}$ (see Definition 2.4) we deduce that $\mathrm{d} \boldsymbol{t}^{\prime} \notin \mathcal{C}_{p}^{\prime \infty}$ for $p \in \bar{H} \cap \mathbb{X}_{a}^{\infty}$, where $H \in \Omega^{\prime}, \boldsymbol{t}^{\prime}:=\boldsymbol{t}_{\mid \bar{H} \times \mathbb{C}}$ and $\mathcal{C}^{\prime \infty}$ is the space of Definition 2.3 starting with the restriction $f_{\mid H}$ instead of $f$. This implies that $a \notin N K_{\infty}\left(f_{\mid H}\right)$.

By taking $H \in \Omega^{\prime} \cap \Omega_{f, a}$ we get in addition that $a \notin J_{f}\left(\operatorname{Sing} f_{\mid H}\right)$. We denote $f_{1}:=f_{\mid H}$.

Now, if $k=2$ in our first assumption at point (b), we may apply the reasoning (a) to $f_{1}$ in place of $f$ and obtain $a \in N K_{\infty}\left(f_{1}\right)$, hence a contradiction.

In case $k>2$, after applying the slicing process (b) exactly $k-2$ more times, namely successively to $f_{1}, \ldots, f_{k-2}$, we arrive to the similar contradiction for $f_{k-1}$.

The inclusion " $\subset$ ". Let $a \in \boldsymbol{t}(B)$ be an asymptotic non-regular value such that the set of $t$-singularities in $\mathbb{X}_{a}^{\infty}$ is not isolated. More precisely, according to Definition 2.5, this set is equal to $\mathbb{X}_{a}^{\infty} \cap \operatorname{Sing}^{\infty} f$. From the remark after Definition 2.5, it follows that $\mathbb{X}_{a}^{\infty} \cap \operatorname{Sing}^{\infty} f$ is an algebraic set. Let therefore $k:=\operatorname{dim} \mathbb{X}_{a}^{\infty} \cap \operatorname{Sing}^{\infty} f$ be its dimension, where $k>0$ by our assumption $\operatorname{dim} B>0$. We show how to reduce $k$ one by one until zero.

For that we use two facts:

(a). From the above proof of the first inclusion we extract the fact that if $\mathrm{d} \boldsymbol{t} \notin \mathcal{C}_{p}^{\infty}$ then $\mathrm{d} \boldsymbol{t}^{\prime} \notin \mathcal{C}_{p}^{\prime \infty}$, for any $H \in \Omega^{\prime}$, where $\boldsymbol{t}^{\prime}:=\boldsymbol{t}_{\mid \bar{H} \times \mathbb{C}}$.

(b). Moreover, by a Bertini type argument ${ }^{1}$, there exists a Zariski-open set $\Omega^{\prime \prime} \subset \check{\mathbb{P}}^{n-1}$ such that if $H \in \Omega^{\prime \prime}$ then $H \times \mathbb{C}$ is transversal to any stratum $\mathcal{W}_{i} \subset \mathbb{X}^{\infty}$ of the Whitney stratification except at finitely many points.

For some $H \in \Omega^{\prime} \cap \Omega^{\prime \prime}$ we consider the restriction $f_{\mid H}$ and the space similar to $\mathbb{X}$ defined at (5) attached to the polynomial function $f_{\mid H}$, which we denote by $\mathbb{Y}$. These two facts imply the equality:

$$
\operatorname{dim}\left(\mathbb{Y}_{a}^{\infty} \cap \operatorname{Sing}^{\infty} f_{\mid H}\right)=\operatorname{dim}\left(\mathbb{X}_{a}^{\infty} \cap \operatorname{Sing}^{\infty} f\right)-1
$$

as long as $k>0$ (which is our assumption). This shows the reduction to $k-1$.

We thus continue to slice by generic hyperplanes and lower one by one the dimension of the set $\operatorname{Sing}^{\infty} f$ until we reach zero, thus we slice a total number of $k$ times. The restriction of $f$ to these iterated slices identifies to the restriction $f_{k}$ defined in the Introduction.

After this iterated slicing we have $f_{k}$ with a nonempty set of isolated $t$-singularities at infinity over $a$, each of which are detected by the horizontal part of the polar curve $\overline{\Gamma\left(x_{k+1}, f_{k}\right)}$, like shown in the first part of the above proof. We therefore get $a \in$ $J_{f}\left(H \Gamma\left(x_{k+1}, f_{k}\right)\right.$.

\footnotetext{
${ }^{1}$ based on the fact that the relative conormal $T_{\boldsymbol{t}_{\mid \mathcal{W}_{i}}}^{*}$ is of dimension $n-1$, the same as $\check{\mathbb{P}}^{n-1}$.
} 
Altogether this shows the inclusion: $N K_{\infty}(f) \subset \bigcup_{i=1}^{n-1} J_{f}\left(H \Gamma\left(x_{i}, f_{i-1}\right)\right) \backslash J_{f}(\operatorname{Sing} f)$. Our proof of Theorem 1.1 is now complete.

3.1. Proof of Corollary 1.2. We estimate the number of Malgrange non-regular values $K_{\infty}(f)$ given by Theorem 1.1. Let us fix a generic system of coordinates $\left(x_{1}, \ldots, x_{n}\right)$. The following equations:

$$
\frac{\partial f_{d}}{\partial x_{2}}=0, \ldots, \frac{\partial f_{d}}{\partial x_{n}}=0
$$

define the algebraic set $\Gamma\left(x_{1}, f\right) \cup \operatorname{Sing} f \subset \mathbb{C}^{n}$ of degree $(d-1)^{n-1}$. Therefore, if nonempty, $\Gamma\left(x_{1}, f\right)$ is a curve of degree $\leq(d-1)^{n-1}$. After Bezout, the curve $\overline{\Gamma\left(x_{1}, f\right)}$ will meet a non-degenerate hyperplane, and in particular the hyperplane at infinity, at a number of points which is bounded from above by $(d-1)^{n-1}-\sum_{i=1}^{r} d_{i}$. Repeating this procedure after successively slicing by general hyperplanes like explained in the above proof, we finally add up the numbers of solutions. This gives the following sum:

$$
(d-1)^{n-1}+(d-1)^{n-2}+\cdots+(d-1)=\frac{(d-1)^{n}-1}{d-2}
$$

to which we have to substract the sums of degrees of the positive dimensional irreducible components of Sing $f$ and their successive slices. It follows that we substract the degree $d_{i}$ a number of $\operatorname{dim} S_{i}$ times which corresponds to the number of times we slice $S_{i}$ and drop its dimension one-by-one until we reach dimension 0. This proves Corollary 1.2.

3.2. New bound for the number of atypical values at infinity. In [JK2, Corollary 1.1] one finds the following upper bound for Malgrange non-regular values:

$$
\# K_{\infty}(f) \leq \frac{d^{n}-1}{d+1} .
$$

Our estimation (2) yields to the following one for $K_{\infty}(f)$ :

$$
\# K_{\infty}(f) \leq \frac{(d-1)^{n}-1}{d-2}-\sum_{i=1}^{r} d_{i} \operatorname{dim} S_{i}+r
$$

This is somewhat sharper than (9). Both have the highest degree term $d^{n-1}$ and the coefficient of the term $d^{n-2}$ in our formula is smaller for high values of $n$.

\section{The NON-PROperness SET AND THE GENERALIZED NOETHER LEMMA}

In this section we give the preliminary material which will lead to the definition in $§ 5$ of the "super-polar curve".

If $f: X \rightarrow Y$ is a dominant, generically finite polynomial map of smooth affine varieties, we denote by $\mu(f)$ the number of points in a generic fiber of $f$. If $\{x\}$ is an isolated component of the fiber $f^{-1}(f(x))$, then we denote by mult $x(f)$ the multiplicity of $f$ at $x$.

Let $X, Y$ be affine varieties, recall that a mapping $f: X \rightarrow Y$ is not proper at a point $y \in Y$ if there is no neighborhood $U$ of $y$ such that $\left.f^{-1}(\bar{U})\right)$ is compact. In other words, $f$ is not proper at $y$ if there is a sequence $x_{l} \rightarrow \infty$ such that $f\left(x_{l}\right) \rightarrow y$. Let $J_{f}$ denote the set of points at which the mapping $f$ is not proper. The set $J_{f}$ has the following properties (see [Jel1], [Jel2], [Jel3]): 
Theorem 4.1. Let $X \subset \mathbb{C}^{k}$ be an irreducible variety of dimension $n$ and let $f=$ $\left(f_{1}, \ldots, f_{m}\right): X \rightarrow \mathbb{C}^{m}$ be a generically-finite polynomial mapping. Then the set $J_{f}$ is an algebraic subset of $\mathbb{C}^{m}$ and it is either empty or it has pure dimension $n-1$. Moreover, if $n=m$ then

$$
\operatorname{deg} J_{f} \leq \frac{\operatorname{deg} X\left(\prod_{i=1}^{n} \operatorname{deg} f_{i}\right)-\mu(f)}{\min _{1 \leq i \leq n} \operatorname{deg} f_{i}}
$$

In the case of a polynomial map of normal affine varieties it is easy to show the following:

Proposition 4.2. Let $f: X \rightarrow Y$ be a dominant and quasi-finite polynomial map of normal affine varieties. Let $Z \subset Y$ be an irreducible subvariety which is not contained in $J_{f}$. Then every component of the set $f^{-1}(Z)$ has dimension $\operatorname{dim} Z$, and if $g$ denotes the restriction of $f$ to $f^{-1}(Z)$, then

$$
J_{g}=J_{f} \cap Z
$$

Proof. By the Zariski Main Theorem in version of Grothendieck, there is an affine variety $\bar{X}$, which contains $X$ as a dense subset and a regular finite mapping $F: \bar{X} \rightarrow Y$ such that $F_{\mid X}=f$. Since the mapping $F$ is finite, all components of $F^{-1}(Z)$ have dimension $\operatorname{dim} Z$. Now the condition $Z \not \subset J_{f}$ implies that all components of $f^{-1}(Z)$ have dimension $\operatorname{dim} Z$. Let $S:=\bar{X} \backslash X$. Observe that $J_{f}=F(S)$. Moreover, $J_{g}=F\left(S \cap F^{-1}(Z)\right)=F(S) \cap Z$.

Let $M_{m}^{n}$ denotes the set of all linear forms $L: \mathbb{C}^{m} \rightarrow \mathbb{C}^{n}$. We need the following result, which is a modification of [Jel5, Lemma 4.1]:

Proposition 4.3. (Generalized Noether Lemma)

Let $X \subset \mathbb{C}^{m}$ be an affine variety of dimension $n$. Let $A \subset \mathbb{C}^{m}$ be a line and $B \subset X$ be a subvariety such that $A \not \subset B$. Let $x_{1}: \mathbb{C}^{m} \rightarrow \mathbb{C}$ be a linear projection and assume that $x_{1}$ is non-constant on $X$ and on $A$. Let $a_{1}, \ldots, a_{s} \in A \cap X$ be some fixed set of points.

There exist a Zariski open dense subset $U \subset M_{m}^{n-1}$ such that for every $(n-1)$-tuple $\left(L_{1}, \ldots, L_{n-1}\right) \in U$ the mapping $\Pi=\left(x_{1}, L_{1}, \ldots, L_{n-1}\right): X \rightarrow \mathbb{C}^{n}$ satisfies the following conditions:

(a) the fibers of $\Pi$ have dimension at most one,

(b) there is a polynomial $\rho \in \mathbb{C}\left[t_{1}\right]$ such that

$$
J_{\Pi}=\left\{\left(t_{1}, \ldots, t_{n}\right) \in \mathbb{C}^{n} \mid \rho\left(t_{1}\right)=0\right\},
$$

(c) $\Pi(A) \not \subset \Pi(B)$,

(d) all fibers $\Pi^{-1}\left(\Pi\left(a_{i}\right)\right), i=1, \ldots, s$ are finite and non-empty.

Proof. For any $Z \subset \mathbb{C}^{m}$, denote by $\tilde{Z}$ the projective closure of $Z$ in $\mathbb{P}^{m}$, and let $H^{\infty}$ denote the hyperplane at infinity. Then $\operatorname{dim} \tilde{X} \cap H^{\infty}=n-1$.

Hence there is a non-empty Zariski open subset $U_{1} \subset M_{m}^{n-1}$ of $(n-1)$-tuples of linear forms such that for any $L=\left(l_{1}, \ldots, l_{n-1}\right) \in U_{1}$ we have $\operatorname{dim} \tilde{X} \cap H^{\infty} \cap \operatorname{ker} L \leq 0$.

Let $l_{n}$ be a general linear form. Since the $(n+1)$ linear forms $\left(x_{1}, l_{1}, \ldots, l_{n}\right)$ are algebraically dependent on $X$, there exists a non-zero polynomial $W \in \mathbb{C}\left[T, T_{1}, \ldots, T_{n}\right]$ such that we have $W\left(x_{1}, l_{1}, \ldots, l_{n}\right)=0$ on $X$. Let us define: 


$$
L_{i}:=l_{i}-\alpha_{i} l_{n}, \text { for } i=1, \ldots, n-1 ; \alpha_{i} \in \mathbb{C}^{*} .
$$

Operating on $W$ the linear change of coordinates $l_{i} \mapsto L_{i}$, for sufficiently general coefficients $\alpha_{i} \in \mathbb{C}$, we then get a relation:

$$
l_{n}^{N} \rho\left(x_{1}\right)+\sum_{j=1}^{N} l_{n}^{N-j} A_{j}\left(x_{1}, L_{1}, \ldots, L_{n-1}\right)=0,
$$

where $N$ is some positive integer, $\rho$ and $A_{j}$ are polynomials, such that $\rho \not \equiv 0$.

The map $P=\left(x_{1}, L_{1}, \ldots, L_{n-1}, l_{n}\right): X \rightarrow \mathbb{C}^{n+1}$ is finite and proper, since $\left(L_{1}, \ldots, L_{n-1}, l_{n}\right)$ is so. Let $X^{\prime}:=P(X)$ and consider the projection:

$$
\pi: X^{\prime} \rightarrow \mathbb{C}^{n}, \quad\left(x_{1}, \ldots, x_{n+1}\right) \mapsto\left(x_{1}, \ldots, x_{n}\right) .
$$

Note that the mapping $\pi$ has fibers of dimension at most one. From the above constructions it follows that the non-properness locus of the projection $\pi$ is:

$$
J_{\pi}=\left\{\left(t_{1}, \ldots, t_{n}\right) \in \mathbb{C}^{n} \mid \rho\left(t_{1}\right)=0\right\},
$$

for the polynomial $\rho \in \mathbb{C}\left[t_{1}\right]$ defined by the relation (12), since $J_{\pi}$ is precisely the locus of the values of $\left(x_{1}, L_{1}, \ldots, L_{n-1}\right)$ such that the equation (12) has less than $N$ solutions for $l_{n}$, counted with multiplicities.

Let us remark that the genericity conditions on $\left(l_{1}, \ldots, l_{n-1}, l_{n}\right) \in M_{m}^{n}$ and the condition that $\left(\alpha_{1}, \ldots, \alpha_{n-1}\right)$ ensure the non-triviality of the polynomial $\rho$ in (12), yield a constructible subset $S$ of $\mathbb{C}^{n-1} \times M_{m}^{n}$. The algebraic mapping:

$$
\Psi: S \rightarrow M_{m}^{n-1}, \quad\left(\alpha_{1}, \ldots, \alpha_{n-1} ; l_{1}, \ldots, l_{n-1}, l_{n}\right) \mapsto\left(L_{1}, \ldots, L_{n-1}\right)
$$

where $L_{i}$ are defined in (11), has a constructible image $\Psi(S) \subset M_{m}^{n-1}$ which contains $U_{1}$ in its closure, thus $\Psi(S)$ contains a non-empty Zariski-open subset $U_{2}$ of $M_{m}^{n-1}$.

We thus obtain (a) and (b) for $U:=U_{2}$ and for $\Pi:=\pi \circ P$.

Next, let us show that there is a non-empty Zariski open subset included in $U_{2}$ such that condition (c) is also satisfied.

Note that $\operatorname{dim} B \leq n-1$. Moreover, there is a point $a \in A \backslash B$, such that the dimension of $B_{a}:=B \cap x_{1}^{-1}\left(x_{1}(a)\right)$ is $<n-1$. Let $\Lambda \subset \mathbb{C}^{m}$ be the Zariski closure of the cone over $B_{a}$ with vertex $a, C_{a} B_{a}:=\bigcup_{x \in B_{a}} \overline{a x}$, which is of dimension $\leq n-1$. Hence

$$
\operatorname{dim} \tilde{\Lambda} \cap H^{\infty}<n-1 .
$$

Consequently, there is a Zariski open subset $U_{3} \subset U_{2}$ such that for $L=\left(L_{1}, \ldots, L_{n-1}\right) \in U_{3}$ we have $\operatorname{dim} \tilde{\Lambda} \cap H^{\infty} \cap \operatorname{ker} L=\emptyset$. This means that for $\Pi:=\left(x_{1}, L_{1}, \ldots, L_{n-1}\right)$ we have $\Pi(a) \notin \Pi(B)$, which finishes the proof of $(\mathrm{c})$.

Let us finally show that there is an eventually smaller non-empty Zariski open subset $U \subset U_{3}$ such that $(\mathrm{d})$ is satisfied too. Let $D_{i}:=x_{1}^{-1}\left(x_{1}\left(a_{i}\right)\right)$, for $i=1, \ldots, s$. Since $\operatorname{dim} D_{i}=n-1$, the Zariski closure $D$ of $\bigcup_{i=1}^{s} D_{i}$ has dimension $n-1$. Hence

$$
\operatorname{dim} H^{\infty} \cap \tilde{D}<n-1 .
$$


Like in the above argument, there is a Zariski open subset $U \subset U_{3}$ such that for $L=$ $\left(L_{1}, \ldots, L_{n-1}\right) \in U$ we have $\operatorname{dim} \tilde{D} \cap H^{\infty} \cap \operatorname{ker} L=\emptyset$. Consequently, for any $i=1, \ldots, s$, the fiber $\Pi^{-1}\left(\Pi\left(a_{i}\right)\right)$ is finite and non-empty.

Definition 4.4. In the notations of Proposition 4.3, we call base-set of non-properness of linear projections of $X$ with respect to $x_{1}$, the set:

$$
B\left(x_{1}, X\right):=\bigcap_{L \in U} J_{\left(x_{1}, L\right)}
$$

REMARK 4.5. If non-empty, the set $B\left(x_{1}, X\right)$ is a finite union of hyperplanes of the form $\left\{b_{i}\right\} \times \mathbb{C}^{n-1}$, by Proposition $4.3(\mathrm{~b})$.

\section{Super-Polar Curve And Proof of TheOrem 1.3}

We have defined at (3) the super-polar curve $\Gamma_{f}(a, b)$ as the Zariski closure of $V\left(g_{1}, \ldots, g_{n-1}\right) \backslash$ $\operatorname{Sing}(f)$, where

$$
g_{i}(a, b):=\sum_{j=1}^{n} a_{i j} \frac{\partial f}{\partial x_{j}}+\sum_{j, k=1}^{n} b_{i j k} x_{k} \frac{\partial f}{\partial x_{j}}, \quad i=1, \ldots, n-1 .
$$

That for general $a_{i j}, b_{i j k} \in \mathbb{C}$ this is indeed a non-degenerate curve follows in particular from the next result, which is equivalent to Theorem 1.3. Let us recall that $H \Gamma_{f}(a, b)$ denotes the horizontal part of $\Gamma_{f}(a, b)$.

Theorem 5.1. There is a Zariski open non-empty set $\Omega$ in the space of parameters $(a, b) \in \mathbb{C}^{n(n+1)}$ such that:

(a) for $(a, b) \in \Omega$ the set $\Gamma_{f}(a, b)$ is a non-empty curve,

(b) $N K_{\infty}(f) \subset J_{f}\left(H \Gamma_{f}(a, b)\right)$.

Proof. Let $\Phi: \mathbb{C}^{n} \rightarrow \mathbb{C} \times \mathbb{C}^{n(n+1)}$ be the polynomial mapping defined by:

$$
\Phi=\left(f, \frac{\partial f}{\partial x_{1}}, \ldots, \frac{\partial f}{\partial x_{n}}, h_{11}, h_{12}, \ldots, h_{n n}\right),
$$

where $h_{i j}=x_{i} \frac{\partial f}{\partial x_{j}}, i=1, \ldots, n, j=1, \ldots, n$.

Let us observe that $\Phi$ is a birational mapping (onto its image), in particular it is generically finite, since $\Phi$ is injective outside the critical set of $f$.

Let $A:=\mathbb{C} \times\{(0, \ldots, 0)\} \subset \mathbb{C} \times \mathbb{C}^{n(n+1)}$. By the definitions of $K_{\infty}(f)$ and of $\Phi$, we have the equality:

$$
K_{\infty}(f)=A \cap J_{\Phi}
$$

where $J_{\Phi}$ denotes the set of points at which the mapping $\Phi$ is not proper. Recall that $K_{\infty}(f)$ is finite, hence the set $A \cap J_{\Phi}$ is finite too.

Let $X:=\overline{\Phi\left(\mathbb{C}^{n}\right)} \subset \mathbb{C} \times \mathbb{C}^{n(n+1)}$ and $B:=J_{\Phi}$. Let $B\left(x_{1}, X\right)$ be a base-set of nonproperness of linear projections of $X$ with respect to $x_{1}$ (cf Definition 4.4).

In the following we identify the target $\mathbb{C}$ of $f$ with the line $A \subset \mathbb{C} \times \mathbb{C}^{n(n+1)}$. 
Let then $\left\{p_{1}, \ldots, p_{s}\right\}:=N K_{\infty}(f) \cup\left(B\left(x_{1}, X\right) \cap A\right) \backslash f(\operatorname{Sing} f) \subset A \cap X$. By Proposition 4.3 and using its notations, for general $\left(L_{1}, \ldots, L_{n-1}\right) \in U \subset M_{1+n(n+1)}^{n-1}$, the mapping:

$$
\Pi=\left(x_{1}, L_{1}, \ldots, L_{n-1}\right): X \rightarrow \mathbb{C}^{n}
$$

satisfies the following conditions:

(a) the fibers of $\Pi$ have dimension at most one,

(b) there is a polynomial $\rho \in \mathbb{C}\left[t_{1}\right]$ such that

$$
J_{\Pi}=\left\{\left(t_{1}, \ldots, t_{n}\right) \in \mathbb{C}^{n} \mid \rho\left(t_{1}\right)=0\right\},
$$

(c) $\Pi(A) \not \subset \Pi(B)$,

(d) all fibers $\Pi^{-1}\left(\Pi\left(p_{j}\right)\right), j=1, \ldots, s$ are finite and non-empty.

Let us write $L_{i}=c_{i} x_{1}+l_{i}(a, b), i=1, \ldots, n-1$, where the linear form $l_{i}(a, b)$ does not depend on variable $x_{1}$. Note that:

$$
\Pi(A)=\left\{x \in \mathbb{C}^{n} \mid x_{1}=t, x_{2}=c_{1} t, \ldots, x_{n}=c_{n-1} t, \quad t \in \mathbb{C}\right\} .
$$

For $\Psi:=\Pi \circ \Phi$, we have (see (14)):

$$
\Pi\left(K_{\infty}(f)\right)=\Pi\left(A \cap J_{\Phi}\right) \subset \Pi(A) \cap J_{\Psi}
$$

Let $V:=\left\{y \in \mathbb{C}^{n} \mid \operatorname{dim} \Psi^{-1}(y)>0\right\}$. Since the fibers $\Psi^{-1}\left(\Pi\left(p_{j}\right)\right), j=1, \ldots, s$, are finite and non-empty we have $\Pi\left(p_{j}\right) \notin \bar{V}$ for $j=1, \ldots, s$. So let $S$ be a hypersurface in $\mathbb{C}^{n}$ which contains $\bar{V}$ but does not contain the set of points $\left\{\Pi\left(p_{1}\right), \ldots, \Pi\left(p_{s}\right)\right\}$ and let

$$
R:=S \cup\left\{y \in \mathbb{C}^{n} \mid \prod_{c \in \Pi(f(\operatorname{Sing} f))}^{r}\left(y_{1}-c\right)=0\right\} .
$$

With these notations, the mapping

$$
\Psi^{\prime}: \mathbb{C}^{n} \backslash \Psi^{-1}(R) \rightarrow \mathbb{C}^{n} \backslash R, \quad x \mapsto \Psi(x)
$$

is quasi-finite, and moreover $\Pi\left(N K_{\infty}(f)\right) \subset J_{\Psi^{\prime}}$.

Let $\Gamma^{\prime}:=\Psi^{\prime-1}(\Pi(A))$. By Proposition 4.2, $\Gamma^{\prime}$ is a curve and $\Pi\left(N K_{\infty}(f)\right)$ is contained in the non-properness set of the mapping $\left.\Psi\right|_{\Gamma^{\prime}}: \Gamma^{\prime} \rightarrow \Pi(A) \backslash R$. Consequently, the set $\Pi\left(N K_{\infty}(f)\right)$ is also contained in the non properness set of the mapping $\Psi$ restricted to $\overline{\Psi^{-1}(\Pi(A) \backslash \Pi(f(\operatorname{Sing} f))}$.

By the definition of $\Psi$ we have $\Psi^{-1}(\Pi(A))=\Phi^{-1}\left(\Pi^{-1}(\Pi(A))\right)$, where:

$$
\Pi^{-1}(\Pi(A))=\left\{x \in X \mid l_{1}(a, b)\left(x_{2}, \ldots, x_{n(n+1)}\right)=0, \ldots, l_{n-1}(a, b)\left(x_{2}, \ldots, x_{n(n+1)}\right)=0\right\} .
$$

Comparing to the definition (13), we see that the set $\overline{\Phi^{-1}\left(\Pi^{-1}(\Pi(A))\right) \backslash \operatorname{Sing}(f)}$ coincides with the super-polar curve $\Gamma_{f}(a, b)$.

The set $\Gamma_{f}(a, b)$ is a curve since it is union of the curve $\overline{\Gamma^{\prime}}$, which actually coincide with the horizontal part $H \Gamma_{f}(a, b)$, and, eventually, some of the one dimensional fibers of $\Psi$.

Let us now consider a linear isomorphism:

$$
T: \mathbb{C}^{n} \rightarrow \mathbb{C}^{n}, \quad\left(x_{1}, \ldots, x_{n}\right) \mapsto\left(x_{1}, x_{2}-c_{2} x_{1}, \ldots, x_{n}-c_{n} x_{1}\right) .
$$

From the above construction we know that $\Pi\left(N K_{\infty}(f)\right) \subset J_{\Psi_{\mid \Gamma^{\prime}}}$. We then have the inclusion $T\left(\Pi\left(N K_{\infty}(f)\right)\right) \subset J_{T \circ \Psi_{\mid \Gamma^{\prime}}}$. But $T \circ \Psi_{\mid \Gamma^{\prime}}$ coincides with $f$ on $\Gamma^{\prime}=H \Gamma_{f}(a, b)$, and 
$T\left(\Pi\left(N K_{\infty}(f)\right)\right)$ coincides with $N K_{\infty}(f)$. This shows the inclusion $N K_{\infty}(f) \subset J_{f}\left(H \Gamma_{f}(a, b)\right)$ and ends the proof of point (b) of our theorem.

\subsection{Proof of Corollary 1.4.}

We use the terminology of the above proof. We have actually shown that if $N K_{\infty}(f) \neq \emptyset$ then the curve $\overline{\Gamma^{\prime}}$ is non-empty, and that the set $N K_{\infty}(f)$ is contained in the nonproperness set of the restriction $f_{\mid \Gamma^{\prime}}$. The curve $\overline{\Gamma^{\prime}}$ is a subset of the super-polar curve $\Gamma_{f}(a, b)$ for general coefficients $a$ and $b$, and moreover, $f$ is constant on all other components of $\Gamma_{f}(a, b)$. By the generalized Bezout Theorem we have $\operatorname{deg} \Gamma_{f}(a, b) \leq d^{n-1}-$ $\sum_{i=1}^{r} d_{i}$, thus $\operatorname{deg} \overline{\Gamma^{\prime}} \leq d^{n-1}-\sum_{i=1}^{r} d_{i}$. Note that the cardinality of the non-properness set of $f_{\mid \Gamma^{\prime}}$ is estimated by the number of these points at infinity of a curve $\Gamma^{\prime}$ which are transformed by $f$ into $\mathbb{C}$. Consequently, the cardinality of the non-properness set of $f_{\mid \Gamma^{\prime}}$ is bounded from above by the number $d^{n-1}-1-\sum_{i=1}^{r} d_{i}$. We can substract 1 in this formula since actually each branch of $\overline{\Gamma^{\prime}}$ intersects the hyperplane at infinity also at the value infinity of $f$. Thus we also have $\# N K_{\infty}(f) \leq d^{n-1}-1-\sum_{i=1}^{r} d_{i}$. Since every connected positive-dimensional component of the critical set Sing $f$ is contained in one fiber of $f$ thus indicates a trivial non-regular value, we obtain:

$$
\# K_{\infty}(f) \leq d^{n-1}-1-\sum_{i=1}^{r}\left(d_{i}-1\right) .
$$

For $n=2$, it turns out that the Malgrange condition can be recovered (see [Ha1], [Ha2], [LO]) by the asymptotic behavior of the derivatives of $f$ only. We thus consider, instead of the mapping $\Phi$ of the proof of Theorem 5.1, the new mapping $\Phi(x, y)=\left(f(x, y), \frac{\partial f}{\partial x}, \frac{\partial f}{\partial y}\right)$. This mapping is generically finite if $N K_{\infty}(f) \neq \emptyset$. In this case, arguing as above we get the last inequalities of our Corollary 1.4.

\section{Algorithm}

We present here a fast algorithm which yields a finite set $S \subset \mathbb{C}$ such that $N K_{\infty}(f) \subset S$, for a given polynomial $f: \mathbb{C}^{n} \rightarrow \mathbb{C}$. By our results, this problem reduces to computing the non-properness set of the mapping $f_{\mid \Gamma}: \Gamma \rightarrow \mathbb{C}$ where $\Gamma$ is a super-polar curve of $f$.

Let us first show how to compute the non-properness set $J_{g}$ of the mapping $g: X \rightarrow \mathbb{C}$, where $X \subset \mathbb{C}^{n}$ is a curve. The following result can be found in [PP]:

Theorem 6.1. If $\mathcal{B}=\left(b_{1}, \ldots, b_{t}\right)$ is the Gröbner basis of the ideal $I \subset k\left[x_{1}, \ldots, x_{n}\right]$ with the lexicographic order in which $x_{1}>x_{2}>\ldots>x_{n}$, then for every $0 \leq m \leq n$ the set $\mathcal{B} \cap k\left[x_{m+1}, \ldots, x_{n}\right]$ is the Gröbner basis of the ideal $I \cap k\left[x_{m+1}, \ldots, x_{n}\right]$.

Corollary 6.2. Consider the ring $\mathbb{C}\left[x_{1}, \ldots, x_{n} ; y_{1}, \ldots, y_{m}\right]$. Let $V \subset \mathbb{C}^{n} \times \mathbb{C}^{m}$ be an algebraic set and let $p: \mathbb{C}^{n} \times \mathbb{C}^{m} \rightarrow \mathbb{C}^{m}$ denote the projection. Assume that $\mathcal{B}$ is a Gröbner basis of the ideal $I(V)$ with the lexicographic order. Then $\mathcal{B} \cap \mathbb{C}\left[y_{1}, \ldots, y_{m}\right]$ is a Gröbner basis of the ideal $I(p(V))$.

Proof. Observe that $I(p(V))=I(V) \cap \mathbb{C}\left[y_{1}, \ldots, y_{m}\right]$ and then use Theorem 6.1. 
Let then $I(X):=\left(h_{1}, \ldots, h_{r}\right)$ be the ideal of our curve $X$. The graph $G \subset \mathbb{C}^{n} \times \mathbb{C}$ of the non-constant mapping $f: X \rightarrow \mathbb{C}$ is given by the ideal $I=\left(h_{i}=0, i=1, \ldots, r ; f(x)-\right.$ z) $\subset \mathbb{C}\left[x_{1}, \ldots, x_{n}, z\right]$.

Let $O$ be the order in $\mathbb{C}\left[x_{1}, \ldots, x_{n}, z\right]$ such that $x_{1}>x_{2}>\ldots>x_{i}>x_{i+1}>\ldots>x_{n}>$ $z$. Let $\mathcal{B}$ denote the Gröbner basis of $I$ with respect to the order $O$. Let $f_{i} \in \mathcal{B} \cap \mathbb{C}\left[x_{i}, z\right]$ be a non-zero polynomial which depends on $x_{i}$. Then:

$$
f_{i}=x_{i}^{n_{i}} a_{0}^{i}(z)+x_{i}^{n_{i}-1} a_{1}^{i}(z)+\ldots+a_{n_{i}}^{i}(z) .
$$

By [Jel1, Prop. 7], [Jel2, Th. 3.10], for our mapping $f: X \rightarrow \mathbb{C}$ we have:

$$
J_{f}=\bigcup_{i=1}^{n}\left\{z \in \mathbb{C} \mid a_{0}^{i}(z)=0\right\} .
$$

With this preparation, we now state the algorithm:

Special case: $\operatorname{Sing}(f)$ is a finite set.

INPUT: the polynomial $f: \mathbb{C}^{n} \rightarrow \mathbb{C}$

(1) choose random coefficients $\alpha_{i}^{k}, \alpha_{i j}^{k}, k=1, \ldots, n-1 ; i, j=1, \ldots, n$.

(2) put $g_{k}=\sum_{j} \alpha_{j}^{k} \frac{\partial f}{\partial x_{j}}+\sum_{i, j} \alpha_{i j}^{k} x_{i} \frac{\partial f}{\partial x_{j}}$.

(3) put $W:=\left(g_{1}, \ldots, g_{n-1}\right) \subset \mathbb{C}\left[x_{1}, \ldots, x_{n}\right]$, if $\operatorname{dim} W>1$ then go back to (1).

(4) compute a Gröbner basis $\mathcal{B}$ of the ideal $I=\left(g_{1}, \ldots, g_{n-1}, f-z\right) \subset \mathbb{C}\left[x_{1}, \ldots, x_{n}, z\right]$ with respect to order $O$ (as defined above).

(5) let $f_{i}=x_{i}^{n_{i}} a_{0}^{i}(z)+x_{i}^{n_{i}-1} a_{1}^{i}(z)+\ldots+a_{n_{i}}^{i}(z) \in \mathcal{B}_{i} \cap \mathbb{C}\left[x_{i}, z\right]$ be a non zero polynomial which depends on $x_{i}$.

(6) let $S:=\bigcup_{i=1}^{n}\left\{z \in \mathbb{C} \mid a_{0}^{i}(z)=0\right\}$. The set $S$ is the non-properness set of the mapping $f$ restricted to $\left.\left\{g_{1}=0, \ldots, g_{n-1}=0\right\}\right)$.

OUTPUT: a finite set $S \subset \mathbb{C}$ such that $N K_{\infty}(f) \subset S$.

In the general case, in order to grip the super-polar curve, we have to remove from the set $\left\{g_{1}=0, \ldots, g_{n-1}=0\right\}$ the singular set $\operatorname{Sing}(f)$. To do this, it is enough to remove the hypersurface $\left\{\sum \beta_{j} \frac{\partial f}{\partial x_{j}}=0\right\}$, where the coefficients $\beta_{j}$ are sufficiently general. Indeed such a hypersurface does contain $\operatorname{Sing}(f)$ but does not contain any component of $\Gamma(a, b)$.

\section{General case:}

INPUT: the polynomial $f: \mathbb{C}^{n} \rightarrow \mathbb{C}$

(1) choose random coefficients $\alpha_{i}^{k}, \alpha_{i j}^{k}, \beta_{i}, k=1, \ldots, n-1, i, j=1, \ldots, n$.

(2) put $g_{k}=\sum_{j} \alpha_{j}^{k} \frac{\partial f}{\partial x_{j}}+\sum_{i, j} \alpha_{i j}^{k} x_{i} \frac{\partial f}{\partial x_{j}}$.

(3) put $h=\sum_{j=1}^{n} \beta_{j} \frac{\partial f}{\partial x_{j}}$.

(4) put $W:=\left(g_{1}, \ldots, g_{n-1}, t h-1\right) \subset \mathbb{C}\left[t, x_{1}, \ldots, x_{n}\right]$; if $\operatorname{dim} W>1$, then go back to (1). 
(5) compute a Gröbner basis $\mathcal{B}$ of the ideal $I=\left(t h-1, g_{1}, \ldots, g_{n-1}, f-z\right) \subset \mathbb{C}\left[t, x_{1}, \ldots, x_{n}, z\right]$ with respect to the order $O$ such that $t>x_{1}>x_{2}>\ldots>\hat{x}_{i}>x_{i+1}>\cdots>$ $x_{n}>>z$.

(6) let $f_{i}=x_{i}^{n_{i}} a_{0}^{i}(z)+x_{i}^{n_{i}-1} a_{1}^{i}(z)+\cdots+a_{n_{l}}^{i}(z) \in \mathcal{B} \cap \mathbb{C}\left[x_{i}, z\right]$ be a non zero polynomial which depends on $x_{i}$.

(7) let $S=\bigcup_{i=1}^{n}\left\{z \in \mathbb{C} \mid a_{0}^{i}(z)=0\right\}$. Here $S$ is the non-properness set of the mapping $f$ restricted to $\left\{g_{1}=0, \ldots, g_{n-1}=0\right\} \backslash\{h=0\}$.

OUTPUT: a finite set $S \subset \mathbb{C}$ such that $N K_{\infty}(f) \subset S$.

REMARK 6.3. The above algorithm is probabilistic (without certification), hence for really random coefficients $\alpha$ and $\beta$ it gives a good subset $S(\alpha, \beta)$, but for some choices it can produce a bad answer. However generically it produces subsets $S(\alpha, \beta)$ which contains $N K_{\infty}(f)$ Therefore in practice we must repeat the algorithm several times and select only the subset $S(\alpha, \beta)$ which contains the same fixed subset all times. The final answer should then be the intersection $S:=\bigcap_{\alpha, \beta} S(\alpha, \beta)$.

At step (5) (and (4) in the isolated singularity case, respectively) we compute Gröbner bases in polynomial rings of at most $n+2$ variables.

It is possible to construct also a version of this algorithm with a certification, however in that case we have to compute Gröbner bases in polynomial rings of $2 n+1$ variables.

REMARK 6.4. A similar algorithm can be constructed for the iterated polar curves method that we use in the first part of our paper; more steps will be needed. We leave the details to the reader.

\section{REFERENCES}

[DRT] L.R.G. Dias, M.A.S. Ruas, M. Tibăr, Regularity at infinity of real mappings and a Morse-Sard theorem. J. Topol. 5 (2012), no 2, 323-340.

[Du] Durfee, Alan H. Five definitions of critical point at infinity. Singularities (Oberwolfach, 1996), 345-360, Progr. Math., 162, Birkhüser, Basel, 1998.

[Fe] M.V. Fedoryuk, The asymptotics of the Fourier transform of the exponential function of a polynomial, Docl. Acad. Nauk 227 (1976), 580-583; Soviet Math. Dokl. (2) 17 (1976), 486-490.

[Gw] J. Gwoździewicz, Ephraim's pencils, Int. Math. Res. Notices 15 (2013), 3371-3385.

[Ha1] Hà Huy Vui, Sur la fibration globale des polynômes de deux variables complexes. C. R. Acad. Sci. Paris Sér. I Math. 309 (1989), no. 4, 231-234.

[Ha2] Hà Huy Vui, Nombres de Lojasiewicz et singularités l'infini des polynômes de deux variables complexes. C. R. Acad. Sci. Paris Sér. I Math. 311 (1990), no. 7, 429-432.

[Jel1] Z. Jelonek, The set of points at which a polynomial map is not proper, Ann. Polon. Math. 58 (1993), 259-266.

[Jel2] Z. Jelonek, Testing sets for properness of polynomial mappings, Math. Ann. 315 (1999), 1-35.

[Jel3] Z. Jelonek, On the Eojasiewicz exponent, Hokkaido Journal of Math. 35 (2006), 471-485.

[Jel4] Z. Jelonek, On asymptotic critical values and the Rabier theorem, Banach Center Publications 65 (2005), 125-133.

[Jel5] Z. Jelonek, On the effective Nullstellensatz, Inventiones Mathematicae 162 (2005), 1-17.

[Jel6] On bifurcation points of a complex polynomial, Proc. AMS. 131, (2003), 1361 - 1367.

[JK1] Z. Jelonek, K. Kurdyka, On asymptotic critical values of a complex polynomial. J. Reine Angew. Math. 565 (2003), 1-11.

[JK2] Z. Jelonek, K. Kurdyka, Reaching generalized critical values of a polynomial, Math. Z. 276 (2014), no. $1-2,557-570$. 
[JK3] Z. Jelonek, K. Kurdyka, Quantitative Generalized Bertini-Sard Theorem for smooth affine varieties, Discrete and Computational Geometry 34, (2005), 659-678.

[JT] Z. Jelonek, M. Tibăr, Bifurcation locus and branches at infinity of a polynomial $f: \mathbb{C}^{2} \rightarrow \mathbb{C}$. Math. Ann. 361 (2015), no. 3-4, 1049-1054.

[LO] Le Van Thanh, M. Oka, Note on estimation of the number of the critical values at infinity. Kodai Math. J. 17 (1994), no. 3, 409-419.

[Pa1] A. Parusiński, A note on singularities at infinity of complex polynomials, in: "Simplectic singularities and geometry of gauge fields", Banach Center Publ. vol. 39 (1997), 131-141.

[Pa2] A. Parusiński, On the bifurcation set of complex polynomial with isolated singularities at infinity, Compositio Math. 97 (1995), 369-384.

[PP] F. Pauer, M. Pfeifhofer, The theory of Gröbner basis, L'Enseignement Mathematique 34 (1988), 215-232.

$[\mathrm{Ph}] \quad$ F. Pham, Vanishing homologies and the $n$ variable saddlepoint method, Arcata Proc. of Symp. in Pure Math., vol. 40, II (1983), 319-333.

[Sa] M. Safey El Din, Testing sign conditions on a multivariate polynomial and applications, Math. Comput. Sci. 1 (2007), no. 1, 177-207.

[ST] D. Siersma, M. Tibăr, Singularities at infinity and their vanishing cycles, Duke Math. Journal 80:3 (1995), 771-783.

[Th] R. Thom, Ensembles et morphismes stratifiés, Bull. Amer. Math. Soc. 75 (1969), 249-312.

[Ti1] M. Tibăr, On the monodromy fibration of polynomial functions with singularities at infinity. C. R. Acad. Sci. Paris Sér. I Math. 324 (1997), no. 9, 1031-1035.

[Ti2] M. Tibăr, Topology at infinity of polynomial mappings and Thom condition, Compositio Math. 111 (1998), 89-109.

[Ti3] M. Tibăr, Asymptotic equisingularity and topology of complex hypersurfaces. Internat. Math. Res. Notices 1998, no. 18, 979-990.

[Ti4] M. Tibăr, Regularity at infinity of real and complex polynomial maps, Singularity Theory, The C.T.C Wall Anniversary Volume, LMS Lecture Notes Series 263 (1999), 249-264. Cambridge University Press.

[Ti5] M. Tibăr, Polynomials and vanishing cycles. Cambridge Tracts in Mathematics, 170. Cambridge University Press, Cambridge, 2007.

[Ve] J.-L. Verdier, Stratifications de Whitney et théorème de Bertini-Sard, Inventiones Math. 36 (1976), 295-312.

Instytut Matematyczny, Polska Akademia Nauk, Śniadeckich 8, 00-956 Warszawa, Poland. E-mail address: najelone@cyf-kr.edu.pl

Mathématiques, UMR 8524 CNRS, Université de Lille 1, 59655 Villeneuve D'AscQ, FRANCE.

E-mail address: tibar@math.univ-lille1.fr 